\title{
Communication Interface Design of Data Acquisition System \\ Based on AT90CAN128
}

\author{
PANG Bin-bin ${ }^{1,2, \text { a }}$, XIANG Yu ${ }^{1, b}$ ZHAO Zi-xu ${ }^{1}$ \\ ${ }^{1}$ Department of Control Engineering of Academy of Armored Forces \\ Engineering, Beijing, China \\ ${ }^{2}$ No.66410 Army \\ apangbinpla@163.com,b519266224@qq.com
}

\begin{abstract}
.
Communication interface of data acquisition based on AT90CAN128 was designed. Given the system hardware frame diagram and program flow charts. 8-channel analog acquisition and 8-channel digital reading were implemented in the system. It succeeded in communicating with the other modules by RS232 and CAN bus. In addition, it monitored the data of communication and fault information online and real time. It was used successfully in the $4 \times 4$ off-road vehicle of electric transmission. The experiment verified the availability of the formula of data acquisition system.
\end{abstract}

Keywords: AT90CAN128; Communication Interface; CAN bus; RS232

\section{Introduction}

The research background of this article is one type $4 \times 4$ electric drive cross-country vehicle, acquisition the data from accelerator pedal sensor, electric quantity detection sensor, motor rate speed sensor, motor temperature sensor, motor current sensor etc. Achieving the aim of motion control and state monitoring of hole vehicle based on the data and control strategy, the communication port of data acquisition system was designed, a kind of popular distribute data acquisition design program at present applied for the system of this article, realized the information exchange and communication between host machine and each aptitude unit. The characteristic of the data acquisition system communication port are: adopting RS232 bus and CAN bus two kind of way realized information interactive, it can meet the requirements of reliability, flexibility, expandable, simple connect fashion, long distance transmission and real time on line data supervisor. 


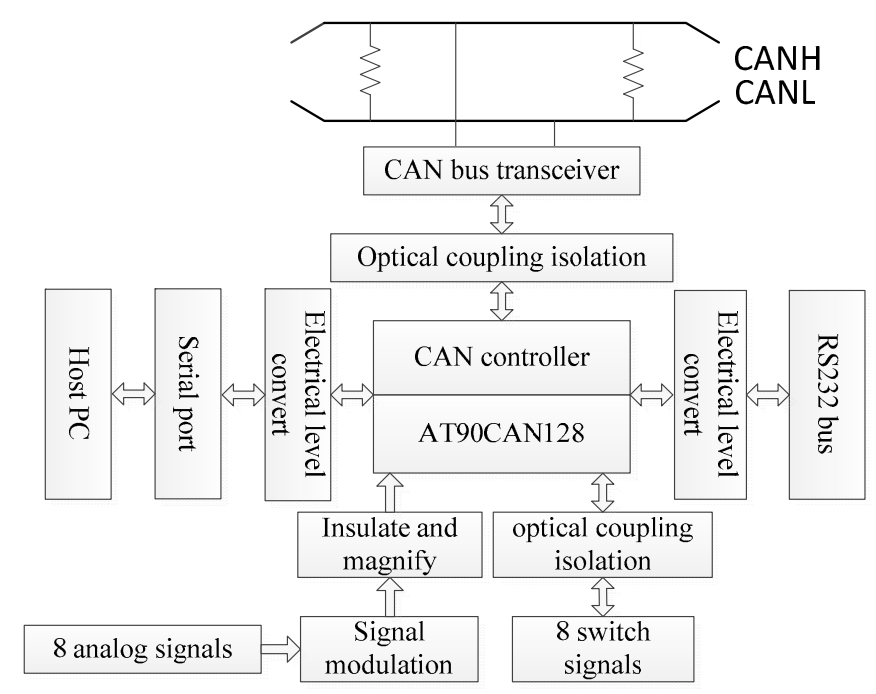

Fig.1 the hardware structure of data acquisition system

\section{System Hardware Circuit Design}

Overall Structure of Hardware System. The hardware system main includes: microprocessor, analog acquisition circuit, digital acquisition circuit, RS232 bus, CAN bus, etc. the microprocessor selects AT90CAN128 produced by ATMEL, the chip has formidable function, it adopt low power dissipation RISC core technology, has following characteristic: $128 \mathrm{~K}$ byte programmable system FLASH, execute cycle of hardware multipliers is two clock cycles, AD transform module, 2 channel serial port, $4 \mathrm{~T} / \mathrm{C}, 53$ current I/O port and CAN controller was integrated[1]. Fig. 1 is overall structure of hardware system.

A/D Transform Circuit. A/D transform circuit adopt A/D transform module which was integrated in AT90CAN123, the module has a 10-bit approximation $\mathrm{ADC}$, the transform time is between 13 us and 260 us, the maximum resolution ratio is $15 \mathrm{kSPS}$, input voltage range is $0 \sim 5 \mathrm{~V}, 810$-bit accuracy ADC input channels, 7 difference input channels, 2 gain selectable of 10x and 200x difference input channels. The module could working in once transform mode or continues transform mode, because of it is inner integrated and its circuit is simple, so it can meets the needs of data acquisition in ordinary industry spot, the transform result as follows:

$$
A D C=\frac{V_{I N} \times 1024}{V_{R E F}}
$$

(1)

In the formula, $V_{I N}$ is input voltage, $V_{R E F}$ is reference voltage, and reference voltage can use inner $2.56 \mathrm{~V}$ reference voltage or outer $5 \mathrm{~V}$ reference voltage. If the 
required accuracy is not exceed 8-bit, it just needs read the high 8-bit of the data in the ADCH register. Signal conditioning circuit mainly use op-amp circuit make up ratio circuit and filtering circuit, adopting liner optical coupling LOC111 to realize liner isolation of signals.

RS-232 Bus Transforms Circuit. The circuit is mainly to complete the scene data transmission and data formats conversion, 2-way serial ports pins of AT90CAN128 connected to corresponding pins of MAX232, when AT90CAN128 needs to transform the data that be collected or received from the CAN bus into RS-232 protocol information and communicate information with field devices or other modules, it can transmit the information to MAX232 through the serial port directly, MAX232 completes data formats converting and communication with other modules.

Multi-channel Switch Signals Input and Output Circuit. This part is completed switch signals input and output, scene fault lights alarm functions. Because data acquisition module of CAN bus can be connected to a variety of analog or digital equipment directly, when the system needs to collect the scene $\mathrm{I} / \mathrm{O}$ information or deal with the results to display, to alarm and other functions, it can by means of pins PA0 PA7 and multiple input and output circuits witch was composed of high-speed optical coupling TLP521.

CAN Bus Communication Hardware Circuit Design.AT90CAN128 microcontroller of ATMEL Corporation is a single chip micyoco witch integrated CAN controller that full compliance with the standard CAN2.0A and 2.0B protocol in Atmel128, using the Mob method to send and receive data, a total of 15 Mobs, they have the same attributes: an identifier 11-bit (2.0A protocol), it also can extends to 29-bit (2.0B); 8-bit data buffer (static allocation); Tx, Rx frame [1].Mob is a CAN message frame descriptor. Including the processing of all information of a CAN message frame. A Mob can be seen as an object used for describes a CAN message, Mob number can be from 0 to 14 , and independent to each other, but when multiple comparisons, the Mob be of lower number would get priority, each Mob has been placed and saved in a page, the page number is the Mob's serial number. CAN bus transceiver circuit consists of three parts: AT90CAN128 microcontroller, high-speed optical coupling 6N137, high-speed transceiver ATA6660. The operating voltage VCC and VDD of optical coupling both sides must adopt isolated power from each other, this system uses $1 \mathrm{~W}$ input and output isolated power modules, achieving the real CAN electrical isolation between nodes through connecting a $0 \mathrm{ohm}$ resistor between different sides ground in the power supply of optical coupling, enhancing the anti-interfering capability of nodes. In this mode, AT90CAN128 microcontroller applies external $8 \mathrm{MHz}$ crystal, CAN bus can realize up to $1 \mathrm{Mb} / \mathrm{s}$ data throughput rate [2-3]. The overall system hardware circuit is shown in Fig.2. 


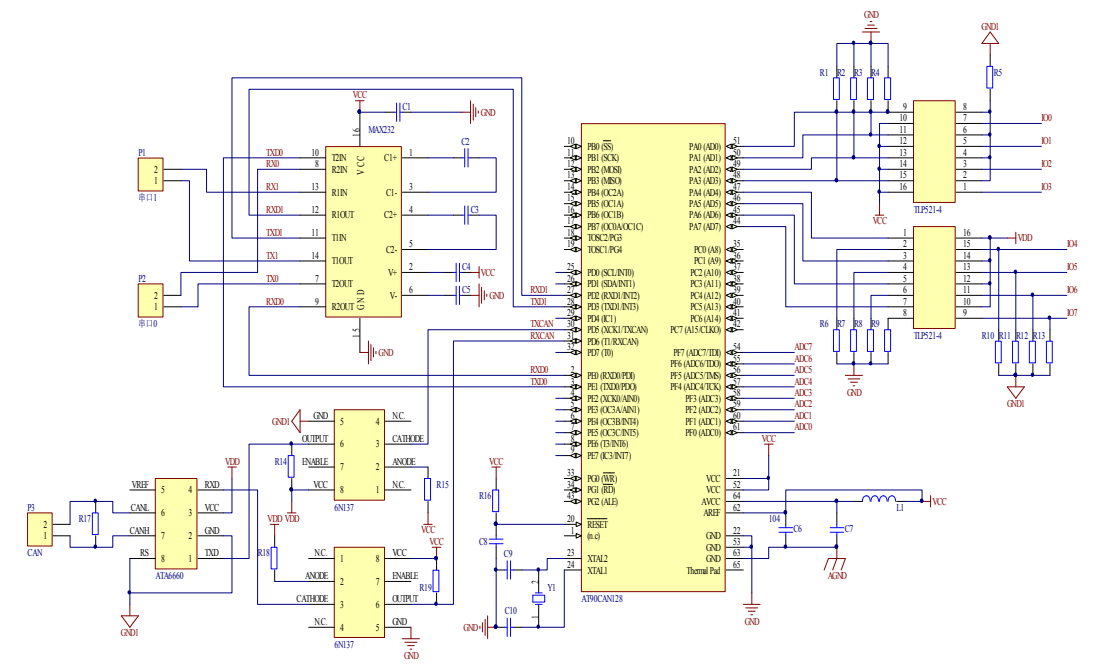

Fig.2 master node system hardware circuit diagram

\section{System Software Design}

Analog Acquisition and Switch Signal Read.AT90CAN128 AD converter module is a 10-bit successive approximation type ADC, 8 AD inputs channel share one sample and hold circuit, so at any time only one way AD channel could acquires data, in order to achieve $8 \mathrm{AD}$ channel analog acquisition, $8 \mathrm{AD}$ input channel gating in order, because of the required data accuracy of system is low, so the delay between each channel is not considered, it mainly applies software programming methods to achieve single conversion mode. The acquisition of switch signals mainly by reading the data of I/O port input pin register PINA, and can achieve I/O port high or low, the process was implemented in the data processing subroutine.

\section{The Master Node Data Sending and Receiving Software Design}

CAN Controller Initialization. Whether the CAN communication is success or not mainly rely on the initialization of CAN controller, initialization is executed in the reset mode, before initialization the soft reset bit of CAN general control register set 1, initialization including: set working patterns, ID Identifier Register (CANIDT) setting, receive screening register (CANIDM) setting, the baud rate setting (CANBT1, CANBT2, CANBT3), message mailboxes Mob control register settings and the interrupt enable register settings etc.

Data Sending. The data sending use query mode, first enable the mailbox that to send data, CAN controller checks the mailbox status register TXOK flag, if the transmit buffer is locked, it would cycle query the status register until the transmit buffer register is released, and fill the sending data filled to CANMSG register in a specific format, by enabling the transmit enable bit of CAN control and data length register $\mathrm{CANCDMOB}$, send mailbox will automatically send messages 
until the CAN control register is empty, during the process of sending a message, send interrupts of CAN controller is disabled, when the transmission is complete, the interrupt flag set 1 .

Data Seceiving.CAN communication data is received must be properly configures acceptance filter register which is made up of four code registers CANIDT and 4 code shielded registers CANIDM, only the data that get through its acceptance filtering would be received. All the bits whose acceptance mask register is 0 , would go through acceptance if the corresponding bit of acceptance code register and CAN message frame must be identical. All the bits whose acceptance mask register is 1,the corresponding bits' acceptance filtering function would be shielded of acceptance code register. CAN communication data receiving can select inquiry mode or interrupt mode, in order to improve the efficiency of data receiving, software programming adopting interrupt mode. Due to AT90CAN128 integrated CAN controller differs from an independent CAN controller, it should be pay more attention when programming the interrupt software, the interrupt sources that can cause CAN controller interrupt triggered are: receive complete interrupt, transmission completion interrupt, the interrupt triggered by a variety of errors, frame buffer full interrupt, the offline settings interrupt, CAN timer overflow interrupt [1].

Main Program Design. The system uses an internal timer overflow interrupt $\mathrm{T} 3$ to designs AD sampling interval, when power is connected, system would initializes I/O port module, USART0 communication module, USART1 communication module, timer T3, CAN controller, AD module, etc. The interrupt program of timer T3 is data acquisition, the main program of serial and CAN send data is shown in fig.3. The interrupt routine will be timed completed $8 \mathrm{AD}$ channels acquisition converter, serial 1 transmit data, CAN transmit data, etc. In order to reduce errors caused by external interference during the analog acquisition, manages to eliminate interference signals were processed, mainly including determine whether exists the data witch error is too large, for repeatedly sampled data using software filter algorithm calculate its average as the sample values and other measures to reduce the system sampling error.

CAN data receive subroutine adopting interrupt mode, monitoring the data of fieldbus, once data errors, or other nodes need to send error and warning messages or other information to the master node, because of the master node receive data interrupt priority than the timer $\mathrm{T} 3$ overflow interrupt, so CAN message register CANMSG receives data firstly and sends to the host computer via the serial port 0 , then the master node sending data normally, it can real-time monitoring other scene nodes. CAN interrupt receive data flow shown in Fig. 4. 


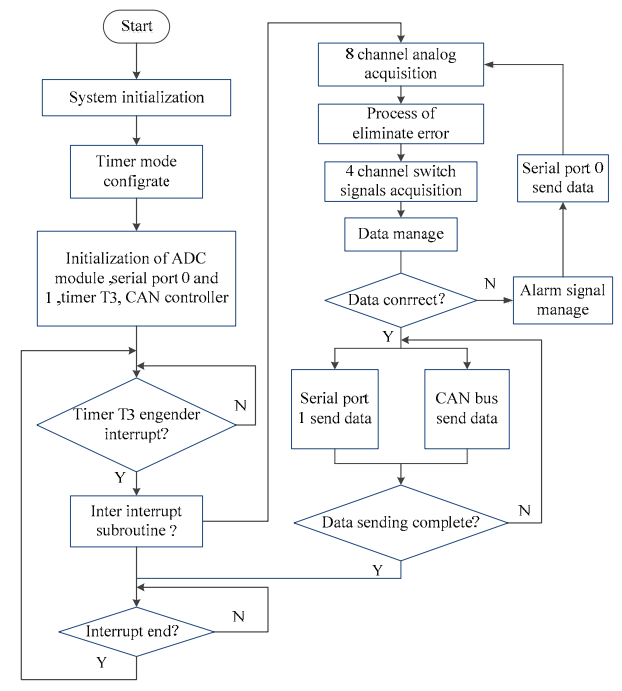

Fig.3 the main program flow

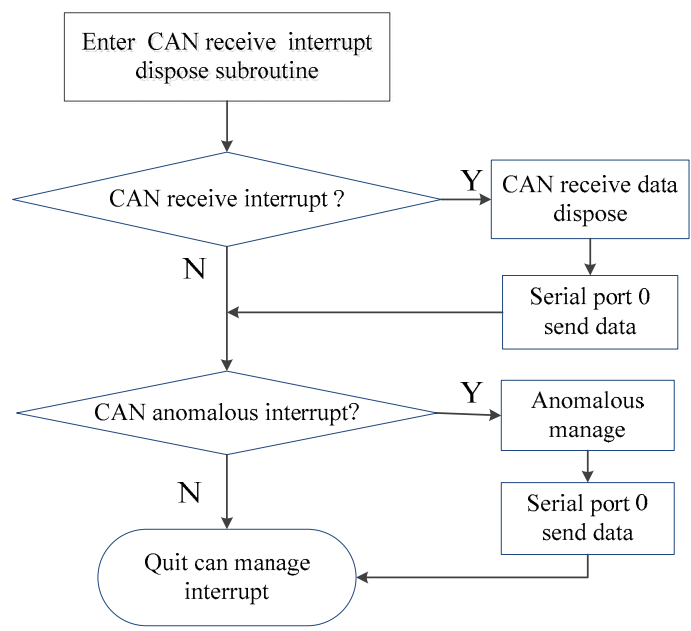

Fig.4 CAN receive data flow 
System Communication Protocol Implementations. In CAN2.0 specification protocol, only specified the physical layer and the data link layer, it doesn' t identify the application layer, so in order to achieve accurate and reliable data transmission, the user must designs CAN application layer protocol properly when designs system software. In CAN communication, the distribution of CAN identifier ID is very important because it determines the priority of information, waiting time, reasonable information filtering efficiency and communication data structures [4]. Taking one type $4 \times 4$ electric drive cross-country vehicle for example, it applies CAN2.0B protocols, data frame has 29 identifiers, data transfer formats are defined as follows: ID. $28 \sim$ ID. 23 are function flag, illustrating the function type of the transmitted information, 000000 means emergency, 000001 means broadcast, 000010 means command, 000011 means surveillance, 0000100 means response; ID.22 ID.21 are multi-frame signs, used for differentiate information frame is multi-frame or single frame; ID.20 ID.13 are destination address; ID. $12 \sim$ ID. 5 are source address; ID. $4 \sim$ ID. 0 are reserved. In CAN massage, node address is represented by the information identifier ID.20 ID.13, specifically allocated as follows: left front motor is 00000001 , right front motor is 00000010 , left after motor is 00000011 , right after the motor is 00000100 .

\section{System experimental results}

Experimental program: collecting HJ9 three-axis hall joystick' s X-axis analog, $\mathrm{Y}$-axis analog, Z-axis and one channel switch signal, analog scope of any one axis is in range of $0-5 \mathrm{~V}$, when joystick at zero site, any axis analog output is $2.34 \mathrm{~V}$. In order to achieves the project application, setting a dead band range $\pm \mathrm{D}$ for the three axes, when the analog input is greater than $2.34+\mathrm{D}$, that is $\mathrm{X}, \mathrm{Y}, \mathrm{Z}$ axis positive direction, defining sign bit is 64 , less than 128 , in order to control convenience, getting more intuitive simulation results, joystick data needs to be necessary processing and conversion, now, imitating it in the Cartesian coordinate system[4]. Assuming the required data range is between - $s$ to $s, s$ is positive, $R$ is the conversion result, the collected axis data $\mathrm{m}$ of the joystick, then the conversion formula as follows:

$$
R=(2 s \times m) / 1024-s
$$

\section{(2)}

Through this formula, converts the data to symmetry position information in the Cartesian coordinate system, i.e., -200 to 200 . Serial experiments using serial debugging assistant, communication baud rate is $9600 \mathrm{kbps}$, CAN communication experiments adopts ZLG USBCAN II , PC software is corresponding ZLGCANTest, the baud rate is $500 \mathrm{kbps}$. 
This data is the measured data when Z-axis is rotated gradually to the maximum meanwhile the $\mathrm{X}, \mathrm{Y}$ axes are within the range of the dead zone, as shown in the figure, the system achieved the goal of data acquisition and two different communication interfaces for data transmission, and it validated the feasibility of the hardware and software.

\section{Conclusion}

Using the new AT90CAN128 microcontroller achieved the goal of scene data acquisition and two different communication interfaces for information transmission with other modules, it also can real-time monitoring $\mathrm{AD}$ data acquisition, and the working state of CAN bus and other scene nodes. The system has been successfully applied to one type $4 \times 4$ electric drive cross-country vehicle, it realized the motion control of the hole vehicle through collecting the HJ9 three-axis hall joystick data, at the same time, achieved real time data monitoring and fault information alarm by displaying the massages in human-machine interface of the vehicle. The system is easy to expand and has a good prospect.

\section{References}

[1] Information on http://www.atmel.com.Rev.4250-CAN-04/05

[2] LI Xiao-jing, ZHANG Kan-yu. Design and implementation of Group Control System for Greenhouse Based on can-bus [J]. Computer Engineering, 2010, 36(1):245-247.

[3] LI Ji-ying. Research on Frequency-shift Send Equipment with Microcomputer [J]. Journal of Lanzhou Jiaotong University, 2007,26(1):32-34.

[4] JIANG Jiang-wen, LIN Yong, HAN Jiang-hong. Analysis and Implementation of Communication Protocol of CAN [J]. Computer Engineering, 2002, 28(2):219-220.

[5] Y. Mishing, in: Diffusion Processes in Advanced Technological Materials, edtied by D. Gupta Noyes Publications/William Andrew Publising, Norwich, NY (2004), in press. 